\title{
Galaxy Evolution in the Infrared: Number Counts and Cosmic Infrared Background
}

\author{
T. T. Takeuchi \\ Division of Particle and Astrophysical Sciences, School of Science, \\ Nagoya University, Nagoya, 464-8602, Japan \\ H. Hirashita, T. T. Ishii, \& K. Yoshikawa \\ Faculty of Science, Kyoto University, Kyoto, 606-8502, Japan
}

\begin{abstract}
Recently reported infrared galaxy number counts and cosmic infrared background (CIRB) measures all suggest that galaxies have experienced a strong evolutionary phase. We statistically estimated the galaxy evolution history from these data. We treated the evolution of galaxy luminosity as a stepwise nonparametric form, in order to explore the most suitable evolutionary history which satisfies the constraint from the CIRB. We found that an order of magnitude increase of the far infrared luminosity at redshift $z=0.75-1.0$ was necessary to reproduce the very high CIRB intensity at $\sim 150 \mu \mathrm{m}$ reported by Hauser et al. (1998). We note that too large an evolutionary factor at high $z$ overpredicts the CIRB intensity around $1 \mathrm{~mm}$. The evolutionary history also satisfies the constraints from galaxy number counts obtained by IRAS, ISO and SCUBA. The rapid evolution of the IR luminosity density required from the CIRB well reproduces the very steep slope of galaxy number counts obtained by ISO. Based on this result and the evolution of optical luminosity density, we quantitatively discuss the contribution of starburst galaxies. In addition, we present the performance of the Japanese IRIS galaxy survey.
\end{abstract}

\section{Discussion}

Martin Harwit: Since your models include metallicity, how do the cumulative metallicities compare to observations?

Tsutomu Takeuchi: We assumed evolution over a range from 0.01 to 1 solar metallicity. 